\title{
Serum SCD163 as a biomarker of adipose tissue inflammation in obstructive sleep apnoea patients: limits and perspectives
}

\begin{abstract}
To the Editor:
Recently, MurPhy et al. [1] demonstrated, through a challenging animal, cellular and human translational approach, that intermittent hypoxia induces a pro-inflammatory activation of adipose tissue macrophages (ATMs), promoting insulin resistance. They confirmed that intermittent hypoxia lowers insulin-mediated glucose uptake in 3T3-L1 adipocytes, and evidenced the interrelationship between inflammation and insulin resistance in the adipose tissue of mice exposed to intermittent hypoxia. They then measured the concentration of serum soluble CD163 (sCD163), an assumed pro-inflammatory biomarker reflecting macrophage activation, in obstructive sleep apnoea (OSA) patients classified according to their BMI and apnoea/hypopnoea index (AHI). This is the first time serum sCD163 has been evaluated in OSA patients, and its association with AHI is promising. With the aim of enhancing its relevance in further studies, we wish to discuss the following points.
\end{abstract}

1) Activation of adipose tissue macrophages (ATMs) towards M1 phenotype in OSA patients. MurPHY et al. [1] made the assumption that circulating sCD163 levels in OSA patients reflect the intermittent hypoxia-dependent polarisation of ATMs towards a M1 phenotype. As appropriately cited, KRAČMEROVÁ et al. [2] showed that circulating sCD163 concentrations are associated with both macrophage content in human adipose tissue, and insulin sensitivity. However, the assumption that serum sCD163 reflects such a polarisation based on the ADAM-17-dependent cleavage, as hypothesised by ETZERODT et al. [3], is unconvincing since only shown in HEK293 cells. Human ATMs are characterised by a high expression of CD163 which, in contrast, is weakly expressed in differentiated M1 macrophages [4]. Therefore, the question arises as to whether a link exists between the ATM/M1 difference in CD163 expression and the increase in serum sCD163 concentration and, if confirmed, to what extent nocturnal intermittent hypoxia impacts this inflammatory activation. An interesting, but technically demanding, approach would be to reproduce, both on control and OSA patients, the experiments from ZEYDA et al. [4] on isolated subcutaneous ATMs, using flow cytometry for ATM/M1/M2 characterisation (including CD163) and quantifying in vitro their cytokine production under stimulating agents, in addition to sCD163 measurements both in serum and in culture supernatants. Besides the ATM/M1 ratio in CD163 expression, ZEYDA et al. [4] showed that ATMs produce higher levels of tumour necrosis factor- $\alpha$, interleukin (IL)-6 and macrophage inflammatory protein- $1 \alpha$ under interferon- $\gamma$ stimulation, and IL-10 under lipopolysaccharide (LPS) stimulation, compared to M1 macrophages. In contrast, under LPS stimulation, M1 macrophages produced high amounts of Rantes, a chemokine not secreted by ATMs. Accordingly, it could be assumed that high circulating Rantes levels observed in obesity hypoventilation syndrome mainly result from an ATM to M1 phenotypic change [5]. Thus, although promising, the association between serum sCD163 and AHI requires further investigation before suggesting sCD163 as a circulating biomarker of ATM activation in OSA patients.

2) Relationship between ATM-specific inflammation and insulin-resistance in OSA patients. MurPHY et al. [1] supported the hypothesis of an intermittent hypoxia-dependent ATM activation through a multiple regression with sCD163 as a dependent variable, from which AHI (or oxygen desaturation index) emerged as the only predictor. Unfortunately, it seems that the authors did not include the homeostasis model assessment resistance index (HOMA-IR) as an independent variable in this model, making it difficult to appreciate if sCD163-based inflammation is related to insulin resistance in these patients. Moreover,

@ERSpublications

Serum sCD163 as a biomarker of adipose tissue inflammation in OSA patients: limits and perspectives http://ow.ly/kgxL30d7RnE

Cite this article as: Monneret D, Bastard J-P. Serum sCD163 as a biomarker of adipose tissue inflammation in obstructive sleep apnoea patients: limits and perspectives. Eur Respir J 2017; 50: 1701038 [https://doi.org/10.1183/13993003.01038-2017]. 
results from univariate regressions between serum sCD163 and body mass index (BMI), waist/hip ratio, HOMA-IR or lipid parameters are lacking, making it difficult to evaluate the relationship between sCD163 and insulin resistance. Indeed, such data could confirm the recent results from CinkajzLové et al. [6], who showed plasma sCD163 as positively associated with BMI, waist/hip ratio, blood glucose, insulin, and HOMA-IR, and negatively associated with high density lipoprotein-cholesterol in a group of subjects including controls, obese and diabetic patients. Besides these statistics, the authors could also provide serum sCD163 mean \pm SD for the BMI- and AHI-based subgroups, which would allow us to compare the ranges from healthy subjects, $\left(334 \pm 25 \mathrm{ng} \cdot \mathrm{mL}^{-1}\right)$, obese $\left(467 \pm 40 \mathrm{ng} \cdot \mathrm{mL}^{-1}\right)$ and diabetic patients $\left(514 \pm 37 \mathrm{ng} \cdot \mathrm{mL}^{-1}\right)$ [6], or from many others pathophysiological contexts [7]. Further studies could include potential correlations of serum sCD163 with more sophisticated anthropometric measurements, such as fat mass.

3) Potential impact of the liver on the relationship between serum sCD163 and OSA severity. Finally, MuRPHy et al. [1] relevantly reminded that, besides adipose tissue disorders, intermittent hypoxia also unbalances hepatic metabolism, favouring steatohepatitis and insulin resistance [8]. Accordingly, an increase in liver enzymes has been shown in OSA $[9,10]$, but still remains controversial. Recently, alanine aminotransferase has been found as positively associated with plasma sCD163 in obese subjects [6]. Thus, although patients with OSA were monitored for liver function, it would have been interesting to present liver enzyme results, and to test their associations with the circulating levels of sCD163.

In conclusion, Murphy et al. [1] highlighted for the first time the potential interest of serum sCD163 as a pro-inflammatory biomarker associated with OSA severity. However, further investigations are needed to confirm the assumption that it reflects the impact of intermittent hypoxia on ATM polarisation towards the M1 phenotype.

Denis Monneret ${ }^{1}$ and Jean-Philippe Bastard ${ }^{2,3,4}$

${ }^{1}$ Dept of Metabolic Biochemistry, La Pitié Salpêtrière-Charles Foix University Hospital (AP-HP), Paris, France. ${ }^{2}$ Service de biochimie et hormonologie, UF biomarqueurs inflammatoires et métaboliques, Hôpital Tenon, AP-HP, Paris, France. ${ }^{3}$ Inserm UMR S938, ICAN, CDR Saint-Antoine, Paris, France. ${ }^{4}$ Sorbonne Universités, UPMC Univ Paris 6, Paris, France.

Correspondence: Denis Monneret, Dept of Metabolic Biochemistry, La Pitié Salpêtrière-Charles Foix University Hospital (AP-HP), 47-83 Boulevard de l'Hôpital, 75013 Paris, France. E-mail: dmonneret2@gmail.com

Received: May 222017 | Accepted: May 242017

Conflict of interest: None declared.

Acknowledgements: The authors thank Vincent Fitzpatrick for the English rereading.

\section{References}

1 Murphy AM, Thomas A, Crinion SJ, et al. Intermittent hypoxia in obstructive sleep apnoea mediates insulin resistance through adipose tissue inflammation. Eur Respir J 2017; 49: 1601731.

2 Kračmerová J, Rossmeislová L, Kováčová Z, et al. Soluble CD163 is associated with CD163 mRNA expression in adipose tissue and with insulin sensitivity in steady-state condition but not in response to calorie restriction. J Clin Endocrinol Metab 2014; 99: E528-E535.

3 Etzerodt A, Maniecki MB, Møller HJ, et al. Tumor necrosis factor $\alpha$-converting enzyme (TACE/ADAM17) mediates ectodomain shedding of the scavenger receptor CD163. J Leukoc Biol 2010; 88: 1201-1205.

4 Zeyda M, Farmer D, Todoric J, et al. Human adipose tissue macrophages are of an anti-inflammatory phenotype but capable of excessive pro-inflammatory mediator production. Int J Obes (Lond) 2007; 31: 1420-1428.

5 Borel JC, Roux-Lombard P, Tamisier R, et al. Endothelial dysfunction and specific inflammation in obesity hypoventilation syndrome. PLoS One 2009; 4: e6733.

6 Cinkajzlová A, Lacinová Z, Kloučková J, et al. An alternatively activated macrophage marker CD163 in severely obese patients: the influence of very low-calorie diet and bariatric surgery. Physiol Res 2017; in press.

7 Zhi Y, Gao P, Xin X, et al. Clinical significance of sCD163 and its possible role in asthma (Review). Mol Med Rep 2017; 15: 2931-2939.

8 Almendros I, García-Río F. Sleep apnoea, insulin resistance and diabetes: the first step is in the fat. Eur Respir J 2017; 49: 1700179

9 Tanné F, Gagnadoux F, Chazouillères O, et al. Chronic liver injury during obstructive sleep apnea. Hepatology 2005; 41: 1290-1296.

10 Kallwitz ER, Herdegen J, Madura J, et al. Liver enzymes and histology in obese patients with obstructive sleep apnea. J Clin Gastroenterol 2007; 41: 918-921.

Copyright @ERS 2017

From the author:

I thank D. Monneret and J-P. Bastard for their interest in our manuscript [1] and I could not agree more with their comment that the role of soluble CD163 (sCD163) in obstructive sleep apnoea (OSA) requires further detailed evaluation. As outlined in our article, the measurement of sCD163 in our patient cohort 
followed our results from the in vitro and in vivo studies demonstrating M1 polarisation of adipose tissue macrophages in response to intermittent hypoxia and thus, was not an a priori hypothesis of our study. Nonetheless, the detected independent association of sCD163 with OSA severity is intriguing.

CD163 is a haemoglobin scavenger receptor that is expressed by cells of the monocyte-M2-macrophage lineage [2]. The soluble form of CD163 is present in plasma of healthy individuals [3] but in response to several inflammatory mediators, levels rise acutely due to metalloproteinase-17/tumour necrosis factor- $\alpha$ converting enzyme (ADAM17/TACE)-mediated cleavage [4-6]. sCD163 has emerged as a sensitive biomarker of inflammation and increased levels have been found in numerous pro-inflammatory conditions, i.e. sepsis, hepatitis, rheumatoid arthritis, scleroderma or atherosclerosis [7, 8]. Moreover, sCD163 is increasingly linked with adipose tissue inflammation and is considered a prognostic predictor for the development of type 2 diabetes [9-11]. Taken all this information together, sCD163 is a very attractive target in OSA.

In our cross-sectional study, univariate analysis revealed a significant correlation of sCD163 with all OSA severity parameters but also with body mass index and waist/hip ratio. However, in linear regression, adjusted for anthropometric and demographic parameters, the apnoea/hypopnoea index (AHI) (or oxygen desaturation index, when substituted for AHI) remained the only significant predictor of this response. Hence, these results along with the pre-clinical data generate the hypothesis that SCD163 may serve as a potential pro-inflammatory marker and predictor of metabolic consequences in OSA. However, testing this hypothesis, as acknowledged in our manuscript, warrants further targeted evaluation including large prospective studies.

@ERSpublications

sCD163 in sleep apnoea requires further evaluation http://ow.ly/o3R130d82YZ

Cite this article as: Ryan S. Serum sCD163 as a biomarker of adipose tissue inflammation in obstructive sleep apnoea patients: limits and perspectives. Eur Respir J 2017; 50: 1701182 [https://doi.org/10.1183/ 13993003.01182-2017].

Silke Ryan ${ }^{1,2}$

${ }^{1}$ Pulmonary and Sleep Disorders Unit, St. Vincent's University Hospital, Dublin, Ireland. ${ }^{2}$ School of Medicine, The Conway Institute, University College Dublin, Dublin, Ireland.

Correspondence: Silke Ryan, Dept of Respiratory Medicine, St Vincent's University Hospital, Elm Park, Dublin 4, Ireland. E-mail: silke.ryan@ucd.ie

Received: June 142017 | Accepted: June 162017

Conflict of interest: None declared.

References

1 Murphy AM, Thomas A, Crinion SJ, et al. Intermittent hypoxia in obstructive sleep apnoea mediates insulin resistance through adipose tissue inflammation. Eur Respir J 2017; 49: 1601731.

2 Kristiansen M, Graversen JH, Jacobsen C, et al. Identification of the haemoglobin scavenger receptor. Nature 2001; 409: 198-201.

3 Møller HJ, Peterslund NA, Graversen JH, et al. Identification of the hemoglobin scavenger receptor/CD163 as a natural soluble protein in plasma. Blood 2002; 99: 378-380.

4 Etzerodt A, Maniecki MB, Møller K, et al. Tumor necrosis factor alpha-converting enzyme (TACE/ADAM17) mediates ectodomain shedding of the scavenger receptor CD163. J Leukoc Biol 2010; 88: 1201-1205.

5 Hintz KA, Rassias AJ, Wardwell K, et al. Endotoxin induces rapid metalloproteinase-mediated shedding followed by up-regulation of the monocyte hemoglobin scavenger receptor CD163. J Leukoc Biol 2002; 72: 711-717.

6 Matsushita N, Kashiwagi M, Wait R, et al. Elevated levels of soluble CD163 in sera and fluids from rheumatoid arthritis patients and inhibition of the shedding of CD163 by TIMP-3. Clin Exp Immunol 2002; 130: 156-161.

7 Buechler C, Eisinger K, Krautbauer S. Diagnostic and prognostic potential of the macrophage specific receptor CD163 in inflammatory diseases. Inflamm Allergy Drug Targets 2013; 12: 391-402.

8 Etzerodt A, Moestrup SK. CD163 and inflammation: biological, diagnostic, and therapeutic aspects. Antioxid Redox Signal 2013; 18: 2352-2363.

9 Kračmerová J, Rossmeislová L, Kováčová Z, et al. Soluble CD163 is associated with CD163 mRNA expression in adipose tissue and with insulin sensitivity in steady-state condition but not in response to calorie restriction. J Clin Endocrinol Metab 2014; 99: E528-E535.

10 Møller HJ, Frikke-Schmidt R, Moestrup SK, et al. Serum soluble CD163 predicts risk of type 2 diabetes in the general population. Clin Chem 2011; 57: 291-297.

11 Parkner T, Sorensen LP, Nielsen AR, et al. Soluble CD163: a biomarker linking macrophages and insulin resistance. Diabetologia 2012; 55: 1856-1862. 